\title{
A Novel REliable Method AsSESS HRV For Heart Disease Diagnosis Using Bipolar MVF ALGORITHM
}

\author{
Mazhar B. Tayel and Eslam I AlSaba \\ Electrical Engineering Department, Faculty Of Engineering., Alexandria University, \\ Alexandria, Egypt
}

\begin{abstract}
In a simple words, the heart rate variability (HRV) refers to the divergence in heart complex wave (beatto-beat) intervals. It is a reliable repercussion of many, psychological, physiological, also environmental factors modulating therhythm of the heart. Seriously, the HRV act as a powerful tool for observation the interaction between the sympathetic and parasympathetic nervous systems. However, it has a frequency that is great for supervision, surveillance, and following up the cases. Finally, the generating structure of heart complex wave signal is not simply linear, but also it involves the nonlinear contributions. Those two contributions are totally correlated.

$H R V$ is stochastic and chaotic (stochaotic) signal. It has utmost importance in heart diseases diagnosis, and it needs a sensitive tool to analyze its variability. In early works, Rosenstein and Wolf had used the Lyapunov exponent (LE) as a quantitative measure for HRV detection sensitivity, but the Rosenstein and Wolf methods diverge in determining the main features of HRV sensitivity, while Mazhar-Eslam introduced a modification algorithm to overcome the Rosenstein and Wolf drawbacks.

The present work introduces a novel reliable method to analyze the linear and nonlinear behaviour of heart complex wave variability, and to assess the use of the HRV as a versatile tool for heart disease diagnosis. This paper introduces a declaration for the concept of the LE parameters to be used for HRV diagnosis and proposes a modified algorithm for a more sensitive parameters computation.
\end{abstract}

\section{KEYWORDS}

Lyapunov exponent (LE), stochastic, Chaotic, stochaotic, Sensitive Dependence (SED), Transform domain, Heart Rate Variability (HRV), sympathetic and parasympathetic, Diagnosis, bipolar Mazhar-Eslam Variability Frequency, Variability Frequency, sensitivity.

\section{INTRODUCTION}

In sinus rhythm the HRV mean the temporal variations in (beat-to-beat) intervals corresponding to Heart Rates instant (HRs).

HRV considered a universal instrument to analyze the heart neural control.Also explain the degree of communication between sympathetic and parasympathetic impact on (HRs). Different pattern can describe the fluctuations in HR such as (linear, non-linear pattern), which classified 
into periodical and a periodical oscillation. These patterns can be quantified in time domain using statistical analysis, in order to estimation the RR-fluctuations intervals.

The analysis by spectroscopy for HRV reveals that the presence of two clear frequency bands variations of HR. High and low frequency band in the range vary from $(0.16-0.4) \mathrm{Hz}$ and $(0.04-$ $0.15) \mathrm{Hz}$ respectively. The higher band frequent act as a marker of vagal modulation, and the lower frequent band indicates the overwhelmingly sympathetic tone and barore flex activity $[1,2,3]$. Each of time domain and frequency domain methods were studied assuming that, the signal of HRV are linear [4], although it failed in full quantification the dynamic structure of the HR signals in order to extraction a highly sensitive diagnostic method for HR diseases.

Really, HRV represent a result for both linear and nonlinear fluctuations. Some interference can modify the linear content of the variability, while constant nonlinear fluctuations. Moreover, the reverse may occur: interference, which up till now have been approved to make cardiovascular fluctuations depends on monitoring with linear methods, can just as well modulate the nonlinear fluctuations. This represent a great work in new drug improvement for patients' treatment.

In the present work introduces a novel reliable method to analyze the linear and nonlinear behaviour of heart complex wave variability, and to assess the use of the HRV as a versatile tool for heart disease diagnosis. The introduced new algorithm based on the Mazhar-Eslam algorithm considering whole cases of linear and nonlinear behaviour for the HRV signal and pattern unlike in Wolf and Rosenstein algorithms [5]. It is a Novel Reliable Method to verify the importance of using the modified Mazhar-Eslam algorithm as a precise predicting tool for HRV diagnosis. It allows to analyze the linear and nonlinear behaviour of HRV. Moreover, it can be considered as a computer aided diagnosis (CAD) of HRV diseases.

\section{Heart Rate VARIABILITY PHENOMENON}

It describes the temporally variation in (beat-to-beat) intervals. Limitation of HR diagnosis method depens on many factors like physical, psychological, and surrounding environmental stressors [5] Backsstated that the validity of the autonomic component, using data from a different research study, in which many central and peripheral psycho-physiological observations were combined together while performing one or double duties which had a diverse physical requirements. For independence, sympathetic and parasympathetic nervous system were tested, using different data From various research, the Principles components analysis factors calculated on raw ECG data supported useful information like various autonomic modes of control were not clear in heart beats. The objective was to verify if factors taken out using residual HR as a sign variable validly reflected cardiac sympathetic activity.If the solutions obtained from raw and baseline corrected data were in acquiescencewith each other. This information about the underlying autonomic activity may raise the efficiency for diagnosis of HR(Figure 1 HR variation -control case). 


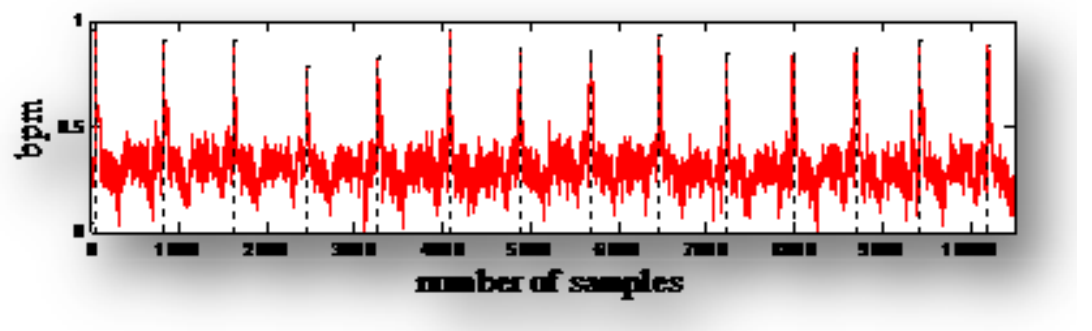

Figure 1.Heart rate variation of a normal subject [6].

\section{NEW (MAZHAR - ESLAM) APPROACH}

Recently, a novel of new approach titled(Mazhar-Eslam) algorithm clarified [5], which used Discrete Wavelet Transform (DWT) considering the merits of DWT over that of FFT. Although the FFT has widely studied, there are still under demands due to its benefits which are not given by FFT. Several points give the priority in choosen DWT instead of FFT. $1^{\text {st }}$ is the hardness of FFT algorithm pruning [5]. Which is not suitable for case of few non-zero inputs located randomly. In simple words, sparse signal does not provide increase to faster algorithm.

The $2^{\text {nd }}$ cause was the accuracy and its speed. Structure part of FFT are one unit and they have equal importance. Thus, it is difficult to choice which part of the FFT structure to delete in case of speed is crucial and error occurring. In simple words, the FFT is conceded a single speed also single accuracy algorithm, which is not suitable for sensitive dependence (SED) cases.

The other reason for not selecting FFT is that there is no built-in noise reduction capacity. Therefore, it is not useful to be used. According to the previous, the DWT is better than FFT especially in the SED calculations used in HRV, because each small variant in HRV indicates the important data and information. Thus, all variants in HRV should be calculated.

The Mazhar-Eslam algorithm depends to some extend on Rosenstein algorithm's strategies [5] to estimate lag and mean period, and uses the Wolf algorithm [5] for calculating the MVF $\left(\Omega_{M}\right)$ except the first two steps, whereas the final steps are taken from Rosenstein's method. Since the $\operatorname{MVF}\left(\Omega_{M}\right)$ measures the degree of the SED separation between infinitesimally close trajectories in phase space, as discussed before, the $\operatorname{MVF}\left(\Omega_{M}\right)$ allows determining additional invariants. Consequently, the Mazhar-Eslam algorithm allows to calculate a mean value for the $\operatorname{MVF}\left(\Omega_{M}\right)$, that is given by

$$
\overline{\Omega_{M}}=\sum_{i=1}^{j} \frac{\Omega_{M_{i}}}{j}
$$

Note that the $\Omega_{M_{i}}$ s contain the largest $\Omega_{M L}$ and variants $\Omega_{M}$ s that indicate to the helpful and important data. Therefore, the Mazhar-Eslam algorithm is a more SED prediction quantitative measure. Therefore, it is robust quantitative predictor for real time, in addition to its sensitivity for all time whatever the period.

Apply the Mazhar-Eslam algorithm to the HRV of the normal case given in fig. 1, it is found that the mean $\operatorname{MVF}\left(\overline{\Omega_{M}}\right)$ as $0.4986 \mathrm{~Hz}$, which is more accurate than Wolf $(0.505 \mathrm{~Hz})$ and Rosenstein $(0.7586 \mathrm{~Hz})$.Figure 2 flowcharts for calculating Mazhar-Eslam MVF algorithm. 
Table (1)shows the different results of the normal case among Mazhar-Eslam, Wolf, and Rosenstein algorithms. The MVF of ideal or optimum case should be $0.5 \mathrm{~Hz}$ as shown in table 1 , thus the accurate one for the normal case should be so close of the range of $0.5 \mathrm{~Hz}$. The table 1 shows how algorithms' MVF close to this range. The Mazhar-Eslam algorithm is the most sensitive as it is the closest on of the 0.5 range. The deviation shown in table one, the Rosenstein is highly deviated as its deviation is 0.2586 . The Wolf success to reach a good sensitive level as its deviation 0.005, but it unlike Mazhar-Eslam algorithm because Mazhar-Eslam algorithm's deviation is 0.0014. Also, from table 1 it is seen that, the Rosenstein algorithm has the lowest SED because of its quite high error( $\mathrm{D}=51.72 \%$ ) comparing to the optimum, while the Wolf algorithm takes a computational place for SED $(\mathrm{D}=1 \%)$. However, the Mazhar-Eslam algorithm shows more sensitivity $(\mathrm{D}=0.28 \%)$ than Wolf algorithm as shown in fig. 3. Besides, the optimum value of variance (Var) for healthy or normal case should tend to 0.25 , thus it is found the Rosenstein is the worst one its variance value is 0.058274 . However the Wolf's variance is acceptable as it is 0.245025 , it is insufficient because HRV depends on the heart and body matter and it needs the most powerful and sensitive tool to analysis it. The variance of Mazhar-Eslam is highly close to optimum as its value is 0.248602 . Therefore, table 1 provide that Mazhar-Eslam algorithm is the most suitable for HRV analysis as discussed previously. The patient case deviation D for normal HRV case is calculated as

Deviation $(D)=\left|\Omega_{M_{\text {normal }}}-\Omega_{M_{\text {case }}}\right|$

the cases percentage deviation is to be calculated as

$D \%=\frac{D}{\text { normal }} \times 100 \%$

and, the variance for algorithms should be calculated as

$\operatorname{var}=\left(\Omega_{M_{\text {normal }}}-D\right)^{2}$

Table 1.The results of the three algorithms for the normal case shown in fig. 1

\begin{tabular}{ccccc}
\hline $\begin{array}{c}\text { method } \\
\text { parameter }\end{array}$ & Optimum & Rosenstein & Wolf & Mazhar-Eslam \\
\hline$\Omega_{M}$ & 0.500000 & 0.758600 & 0.505000 & 0.498600 \\
$\mathrm{D}$ & 0.00000 & 0.258600 & 0.005000 & 0.001400 \\
$\mathrm{D} \%$ & 0.000000 & 51.720000 & 1.000000 & 0.280000 \\
Var & 0.250000 & 0.058274 & 0.245025 & 0.248602 \\
\hline
\end{tabular}

The bar diagram in fig. 4 shows the percentage deviation of the three algorithms. From this figure it is seen that the Mazhar-Eslam algorithm gives the best result as it has the lowest percentage deviation $(\mathrm{D}=14)$. At the same time, when calculating the variance to determine the accurate and best method, Mazhar-Eslam algorithm gives the best value. Figure 5 shows the bar diagram of the variance for normal control case using the HRV for Wolf, and Mazhar-Eslam algorithms. It is clear that the Mazhar-Eslam algorithm is more powerful and accurate than Wolf, because its variance better than Wolf by 0.0036 . This result comes because the Mazhar-Eslam considers all the variability mean frequencies $\overline{\Omega_{M}} \mathrm{~s}$ unlike the Wolf method as it takes only the largest. Each interval of the HRV needs to be well monitored and taken into account because the variant in $\mathrm{HRV}$ is indication of cases. 
International Journal of Biomedical Engineering and Science (IJBES), Vol. 3, No. 1, January 2016

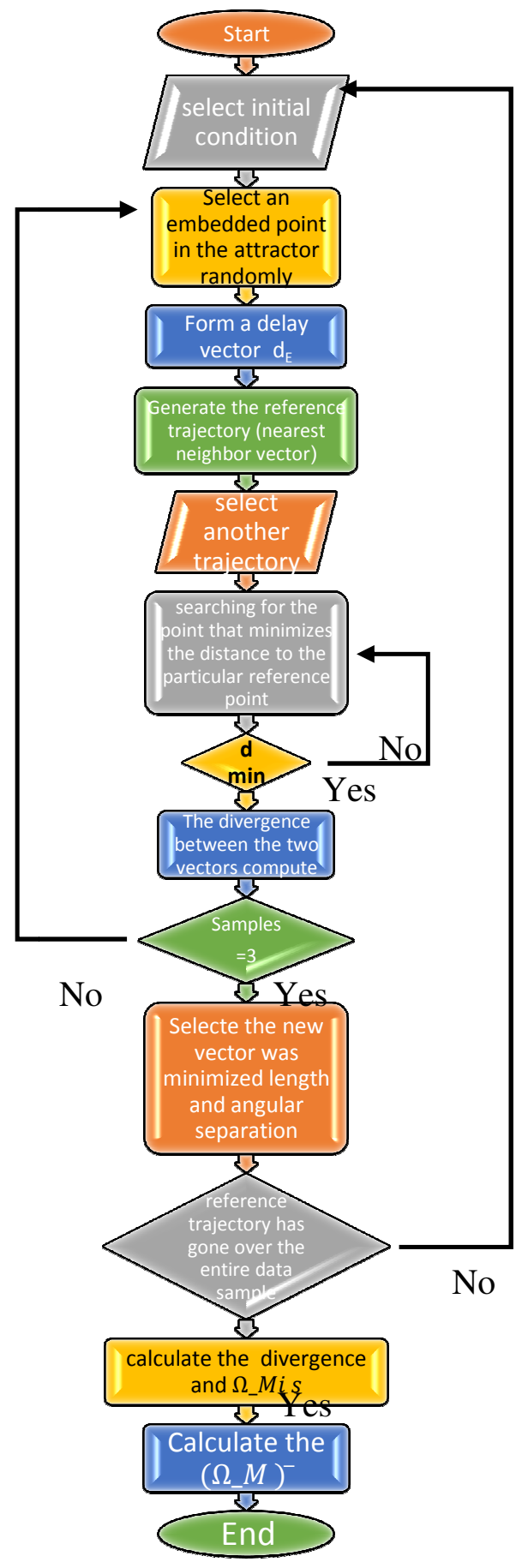

Figure 2.The flowchart of the (Mazhar-Eslam) algorithm.

From the bar diagram in fig. $\mathbf{5}$ it is seen that the Mazahar-Eslamalgorithm is most useful and sensitive comparing to Wolf and Rosenstein algorithms. 
International Journal of Biomedical Engineering and Science (IJBES), Vol. 3, No. 1, January 2016

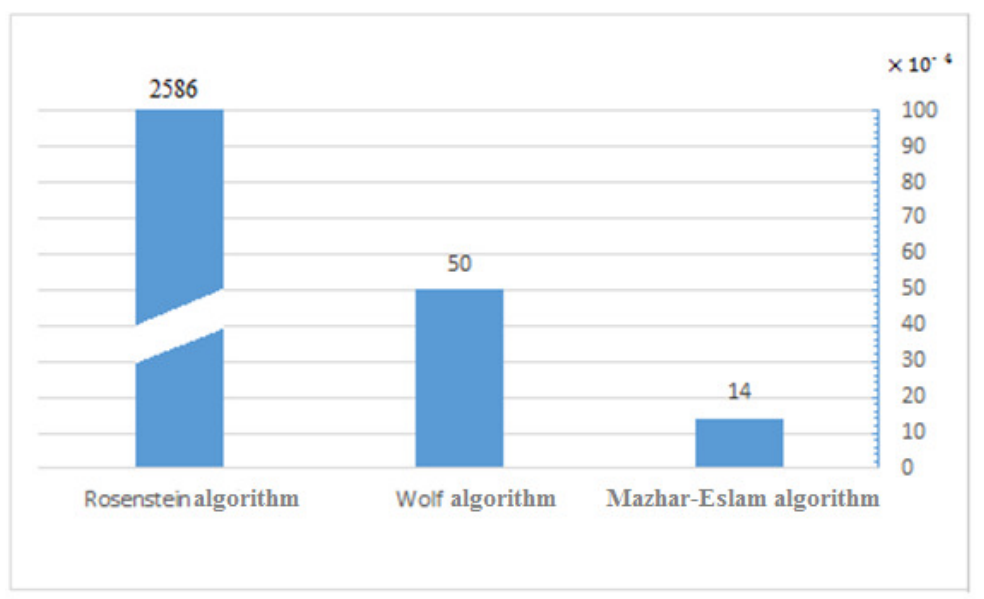

Figure 3.The three algorithms deviation for the normal case in fig. 1.

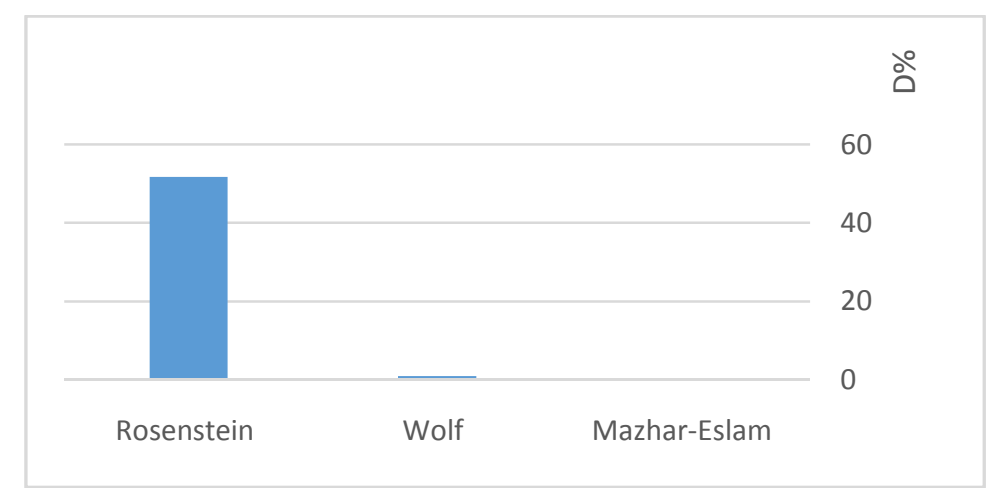

Figure 4.The three algorithms Percentage deviation (D\%) for the normal case fig. 1.

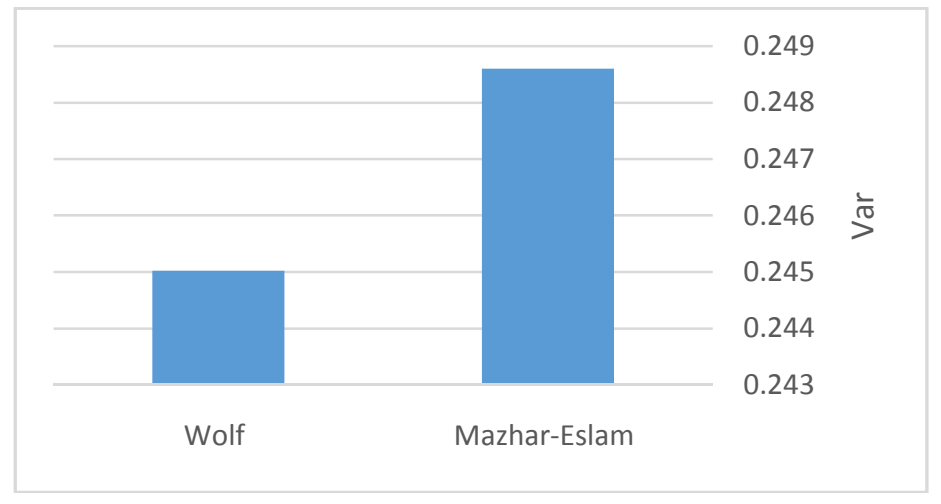

Figure 5.The Variance of Wolf and Mazhar-Eslam algorithm for normal case fig. 1. 
International Journal of Biomedical Engineering and Science (IJBES), Vol. 3, No. 1, January 2016

\section{Bipolar MVf Algorithm}

The MVF " $\Omega_{M}$ "diversity of initially closely trajectories in state-space is connected with folding of them. The presence of a positive part $\operatorname{MVF}\left(\Omega_{M}>0\right)$, for all initial conditions in a restricted dynamical system, is the vastly used definition of deterministic chaos. Thus, to distinguish between periodic signals chaotic and, dynamics the MVF $\Omega_{M}$ are predominantly used. The trajectories of chaotic signals in state-space pursue typical patterns. Nearlydiverge trajectories diverge and converge exponentially, proportional to each other. A negative $\operatorname{MVF}\left(\Omega_{M}<0\right)$ means that the orbit enticesto a settled point or stable periodic orbit. Negative MVFs are distinguishing of non-fogyish systems. Like systems display asymptotic stability. For more stability, the MVF is more negative. When MVF tends to infinity i.e. $\Omega_{M}=-\infty$, it is mean the excessive stable periodicity.

\subsection{BIPOLAR (MAZHAR-ESLAM) VARIABILITY FREQUENCY (BMVF)}

Generally, the HRV for healthy person (normal) seems to be periodic stochaotic signal, while the HRV for a patient is usually a periodic stochaotic signal. Thus, in case will be stable if variability signal (i.e. stochaotic signal) is periodic. Also, it gives an indication of utmost important information. Subsequently, stochaotic signal of the HRV periodicity should be studied and analysed for variability prediction.

It is clear that the MVF is the most suitable and sensitive tool for predicting the HRV. Therefore, in the following it would be used to predict and verify the importance of HRV stochaotic periodicity. Also, it was stated that the positive part indicates case status and the negative part indicates the stability and periodicity. This explains the necessity to consider both polarities (positive and negative) of the MVF, (i.e. $\Omega_{M}>0$ and $\Omega_{M}<0$ ). Also, it was stated that in the present work the stochaotic periodicity and variation in the HRV the negative and positive MVFs $\Omega_{M}$ s should be taken in account. Thus, a new approach to be defined as Bipolar Mazhar-Eslam Variability Frequency method is introduced.

Theoretically speaking, the introduced Bipolar Mazhar-Eslam Variability Frequency (BMVF) calculated from ideal HRV, that comes from ideal Electrocardiograph (ECG) is equal to 0.5 for positive MVF indicating a healthiest case, and is equal to -1 for the negative MVF indicating more HRV stochaotic periodic signal. Thence, the difference of the introduced Bipolar MazharEslam Variability Frequency $\bar{\Omega}_{M_{B}}$ is -0.5 , and around this value, the case is healthy. For the normal (control) case shown in (Fig. 1), the introduced Bipolar Mazhar-Eslam BMVF $\overline{\Omega_{M_{B}}}$ is 0.4986 and -0.9832 . The difference of the BMVF for normal (control) case is -0.4846 , which is very close to -0.5 . Consequently, the introduced Bipolar Mazhar-Eslam Variability Frequency BMVF has a great role in monitoring, predicting and diagnosing the HRV stochaotic signal. The BMVF has ability to monitor and follow up the patient case. It shows the disease by the positive $\operatorname{BMVF}\left(\overline{\Omega_{M_{B}}}>0\right)$ part and shows the periodicity by the negative BMVF $\left(\overline{\Omega_{M_{B}}}<0\right)$ part.

To verify and show the benefits of the BMVF some critical diseases data from the MIT-BIH are used. The table 2 discusses the introduced Bipolar Mazhar-Eslam Variability Frequency BMVF in many different cases from the MIT-BIH and compares it with Mazhar-Eslam, Wolf, and Rosenstein algorithms results. The figures 6 and 7 present the MVF distribution. 
International Journal of Biomedical Engineering and Science (IJBES), Vol. 3, No. 1, January 2016

Table 2.The MVF results of different methods using normal case and the MIT-BIH sample cases

\begin{tabular}{|c|c|c|c|c|c|c|}
\hline \multirow{3}{*}{$\begin{array}{l}\text { 产 } \\
\text { कू }\end{array}$} & \multirow{3}{*}{$\begin{array}{l}\text { Parameter } \\
\text { Method } \\
\text { Case }\end{array}$} & \multicolumn{3}{|c|}{ MVF } & \multicolumn{2}{|c|}{$\overline{\text { BMVF }}$} \\
\hline & & \multirow[t]{2}{*}{ Rosenstein } & \multirow[t]{2}{*}{ Wolf } & \multirow[t]{2}{*}{ "Mazhar-Eslam } & & \\
\hline & & & & & $\overline{\overline{\Omega_{M_{B}}}}>\mathbf{0}$ & $\overline{\Omega_{M_{B}}}<\mathbf{0}$ \\
\hline 1 & Normal & 0.7586 & 0.5050 & 0.4986 & 0.4986 & $\begin{array}{c}-0.9832 \\
\end{array}$ \\
\hline 2 & 101 & 0.2500 & 0.1700 & 0.0830 & 0.0830 & -0.1200 \\
\hline 3 & 102 & 0.1600 & 0.1300 & 0.0530 & 0.0530 & -0.0830 \\
\hline 4 & 104 & 0.2100 & 0.1300 & 0.0700 & 0.0700 & -0.1100 \\
\hline 5 & 106 & 0.2300 & 0.1500 & 0.0770 & 0.0770 & -0.1130 \\
\hline 6 & 107 & 0.2000 & 0.1300 & 0.0667 & 0.0667 & -0.1030 \\
\hline 7 & 109 & 0.2200 & 0.1400 & 0.0733 & 0.0733 & -0.1100 \\
\hline 8 & 111 & 0.2400 & 0.1600 & 0.0800 & 0.0800 & -0.1230 \\
\hline 9 & 112 & 0.2400 & 0.1700 & 0.0800 & 0.0800 & -0.1170 \\
\hline 10 & 115 & 0.2800 & 0.1700 & 0.0930 & 0.0930 & -0.1330 \\
\hline 11 & 117 & 0.2300 & 0.1600 & 0.0770 & 0.0770 & -0.1100 \\
\hline 12 & 118 & 0.2500 & 0.1600 & 0.0833 & 0.0833 & -0.1230 \\
\hline 13 & 119 & 0.2700 & 0.1700 & 0.0900 & 0.0900 & -0.1370 \\
\hline 14 & 121 & 0.2500 & 0.1600 & 0.0840 & 0.0840 & -0.1200 \\
\hline 15 & 122 & 0.2300 & 0.1600 & 0.0770 & 0.0770 & -0.1140 \\
\hline 16 & 123 & 0.2300 & 0.1500 & 0.0770 & 0.0770 & -0.1140 \\
\hline 17 & 124 & 0.2500 & 0.1700 & 0.0840 & 0.0840 & -0.1300 \\
\hline 18 & 200 & 0.2300 & 0.1500 & 0.0770 & 0.0770 & -0.1130 \\
\hline 19 & 203 & 0.2300 & 0.1500 & 0.0770 & 0.0770 & -0.1140 \\
\hline 20 & 212 & 0.2100 & 0.1400 & 0.0700 & 0.0700 & -0.1100 \\
\hline 21 & 221 & 0.2100 & 0.1400 & 0.0700 & 0.0700 & -0.1070 \\
\hline 22 & 230 & 0.2100 & 0.1400 & 0.0700 & 0.0700 & -0.1030 \\
\hline 23 & 231 & 0.2200 & 0.1500 & 0.0740 & 0.0740 & -0.1100 \\
\hline
\end{tabular}
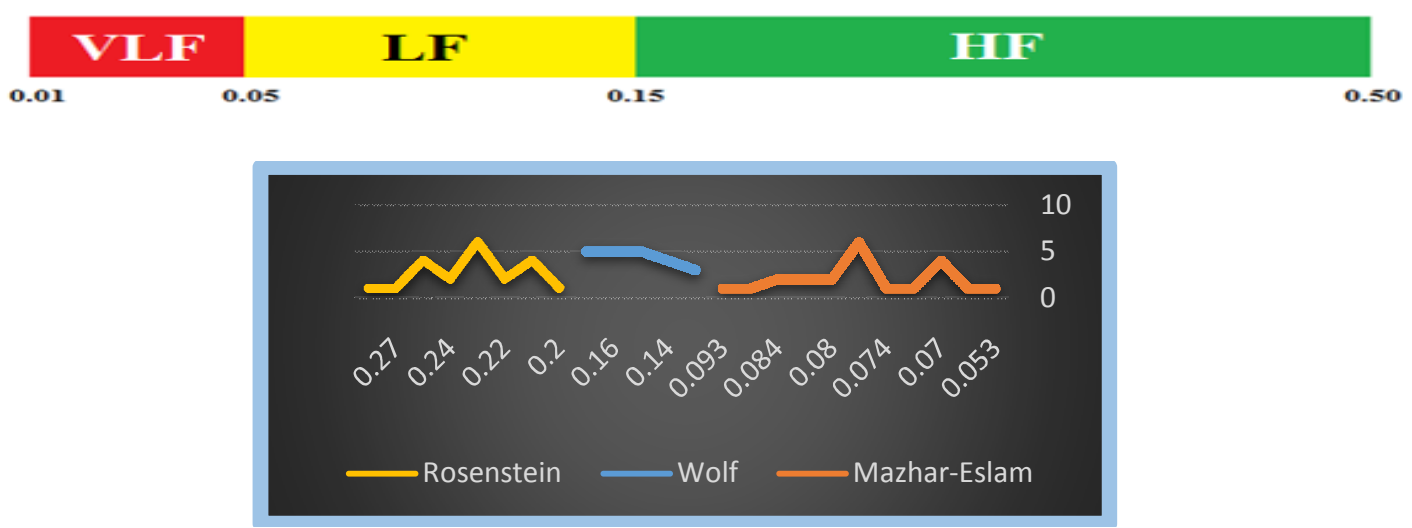

Figure 6.MVF distribution 


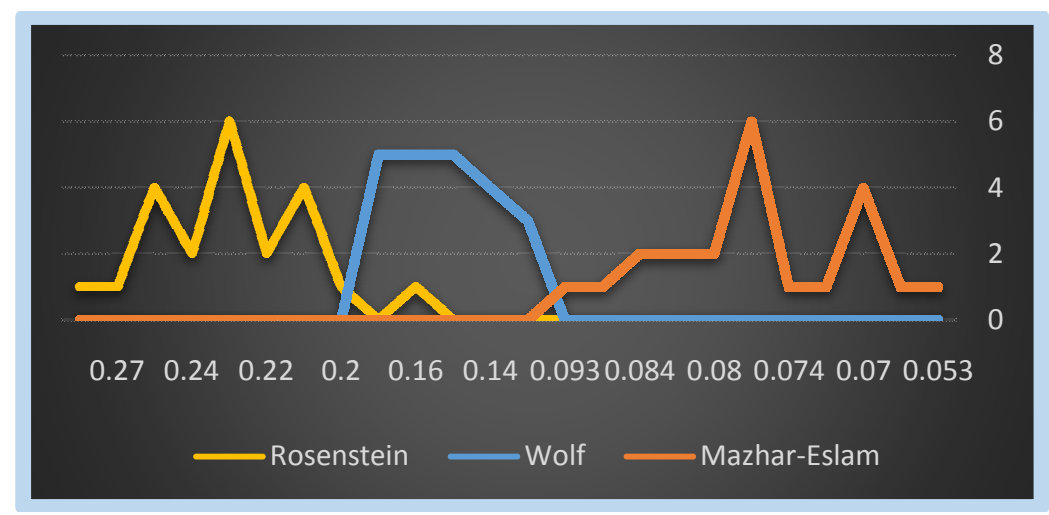

Figure 7.MVF distribution

\subsection{DISCUSSIONS}

To study and analyse table 2, the cases must be arranged related to field study. The results of the previous proposal is based on studding of field is sensitivity dependence (SED) of algorithms is introduced in next.

\subsubsection{StUdy OF SENSITIVITY DEPENDENCE (SED)}

The sensitivity dependence (SED) in prediction of the HRV is so important. Table 2gives the results of computation of MVF for the four algorithms namely: Rosenstein, Wolf, Mazhar-Eslam, and the introduced Bipolar Mazhar-Eslam (BMVF). From this table 2 the results rearranged relative the sensitivity. The next table 3 discusses the SED of three MVF algorithms: Rosenstein, Wolf, and Mazhar-Eslam. The results were rearranged depending on Wolf algorithm results compared to Mazhar-Eslam and Rosenstein algorithms results, since the Wolf distributed has same variability in many difference cases.

The results in table 3 were grouped depend on the Wolf values. This table shows the sensitivity of the three MVF algorithms. The Wolf results show the cases in same area of diseases. Actually, they are different although these have the same main problem like supra ventricular ectopy and ventricular ectopy, they have other diseases. Thus, the BMVF is used to predict any tiny change in HRV that depends on many information and different diseases. However, the BMVF algorithms success to predict sensitively the HRV signal, they have variation in the accuracy and sensitivity. From the table 3, it is clear that the Rosenstein algorithm is unsuitable for predict the critically heart disease cases and the long data set as however its sensitivity. The Rosenstein is sensitive tool but its variation is not small enough to be accepted for HRV prediction because any tiny change should be predicted. The Wolf algorithm scores better accuracy than Rosenstein. Unfortunately, its results are stable in many cases and the HRV needs more sensitive and accurate tool to be predicted. Consequently, the table 5.4 shows that depending on the result and cases status in MIT-BIH medical reports data, the Mazhar-Eslam is the most sensitive and accurate MVF tool for HRV. The figure 8 that presents the bar diagram of MVF algorithms SED and figure 9 summarize the sensitivity dependence (SED) of MVF algorithms. 
International Journal of Biomedical Engineering and Science (IJBES), Vol. 3, No. 1, January 2016

Table 3The SED of MVF algorithms in different algorithms using normal case and the MIT-BIH sample cases.

\begin{tabular}{|c|c|c|c|c|c|c|}
\hline \multirow{3}{*}{ 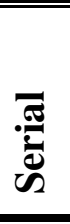 } & \multirow{3}{*}{$\begin{array}{l}\text { Parameter } \\
\text { Method } \\
\text { Case }\end{array}$} & \multicolumn{3}{|c|}{ MVF } & \multirow{2}{*}{\multicolumn{2}{|c|}{ BMVF }} \\
\hline & & \multirow[t]{2}{*}{ Rosenstein } & \multirow[t]{2}{*}{ Wolf } & \multirow[t]{2}{*}{$\begin{array}{l}\text { Mazhar- } \\
\text { Eslam }\end{array}$} & & \\
\hline & & & & & $\overline{\Omega_{M_{B}}}>\mathbf{0}$ & $\overline{\Omega_{M_{B}}}<\mathbf{0}$ \\
\hline 1 & Normal & 0.7586 & 0.505 & 0.4986 & 0.4986 & -0.9832 \\
\hline 2 & 112 & 0.2400 & 0.1700 & 0.0800 & 0.0800 & -0.1170 \\
\hline 3 & 101 & 0.2500 & 0.1700 & 0.0830 & 0.0830 & -0.1200 \\
\hline 4 & 124 & 0.2500 & 0.1700 & 0.0840 & 0.0840 & -0.1300 \\
\hline 5 & 119 & 0.2700 & 0.1700 & 0.0900 & 0.0900 & -0.1370 \\
\hline 6 & 115 & 0.2800 & 0.1700 & 0.0930 & 0.0930 & -0.1330 \\
\hline 7 & 117 & 0.2300 & 0.1600 & 0.0770 & 0.0770 & -0.1100 \\
\hline 8 & 122 & 0.2300 & 0.1600 & 0.0770 & 0.0770 & -0.1140 \\
\hline 9 & 111 & 0.2400 & 0.1600 & 0.0800 & 0.0800 & -0.1230 \\
\hline 10 & 118 & 0.2500 & 0.1600 & 0.0833 & 0.0833 & -0.1230 \\
\hline 11 & 121 & 0.2500 & 0.1600 & 0.0840 & 0.0840 & -0.1200 \\
\hline 12 & 231 & 0.2200 & 0.1500 & 0.0740 & 0.0740 & -0.1100 \\
\hline 13 & 203 & 0.2300 & 0.1500 & 0.0770 & 0.0770 & -0.1140 \\
\hline 14 & 200 & 0.2300 & 0.1500 & 0.0770 & 0.0770 & -0.1130 \\
\hline 15 & 123 & 0.2300 & 0.1500 & 0.0770 & 0.0770 & -0.1140 \\
\hline 16 & 106 & 0.2300 & 0.1500 & 0.0770 & 0.0770 & -0.1130 \\
\hline 17 & 212 & 0.2100 & 0.1400 & 0.0700 & 0.0700 & -0.1100 \\
\hline 18 & 221 & 0.2100 & 0.1400 & 0.0700 & 0.0700 & -0.1070 \\
\hline 19 & 230 & 0.2100 & 0.1400 & 0.0700 & 0.0700 & -0.1030 \\
\hline 20 & 109 & 0.2200 & 0.1400 & 0.0733 & 0.0733 & -0.1100 \\
\hline 21 & 102 & 0.1600 & 0.1300 & 0.0530 & 0.0530 & -0.0830 \\
\hline 22 & 107 & 0.2000 & 0.1300 & 0.0667 & 0.0667 & -0.1030 \\
\hline 23 & 104 & 0.2100 & 0.1300 & 0.0700 & 0.0700 & -0.1100 \\
\hline
\end{tabular}

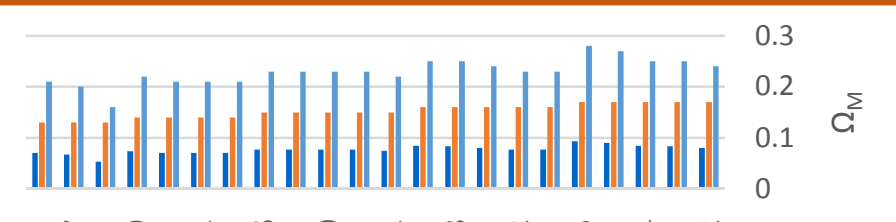

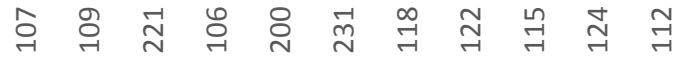

MIT-BIH cases

Rosenstein $\square$ Wolf $\square$ Mazhar-Eslam

Figure 8.Bar diagram for MVF algorithms SED 


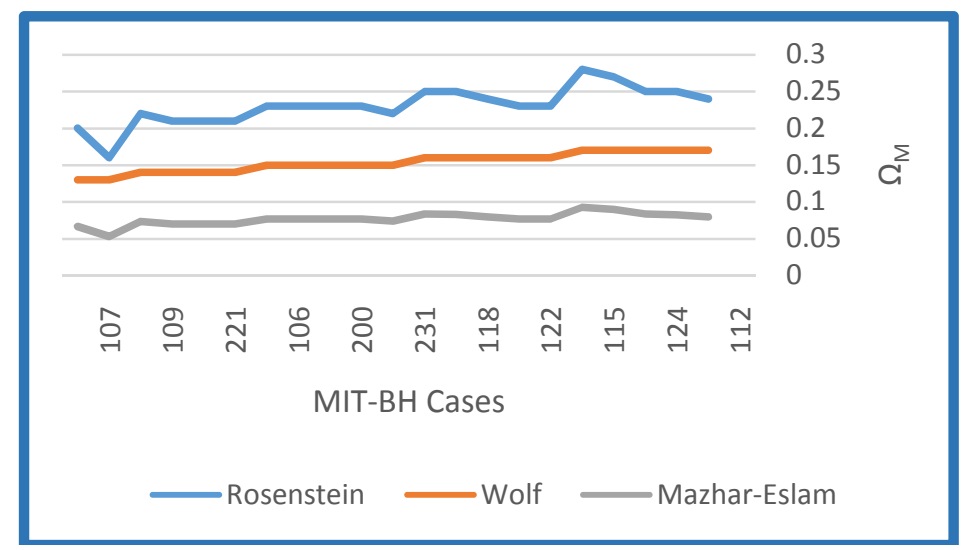

Figure 9.MVF algorithms SED

(Figure 8, and 9) show that the Mazhar-Eslam algorithm is the most sensitive MVF tool. The Mazhar-Eslam results change with a range of diseases but with a sensitive variation. The Rosenstein algorithm is out of comparison because its results have a huge variation for the same disease. The Wolf algorithm is sensitive, but its sensitivity is not enough because it is stable for many cases although they are different. Thence, it is so clear that the Mazhar-Eslam is the best method and as a quantities measure.

\section{CONCLuSions}

Heart Rate Variability (HRV) is presented in manydiseases whatever it related to the heart or not. Besides, it has good prediction class or level and importance in modelling the heart risk. HRV is stochaotic signal that remains highly controversial. In order to have utmost importance, HRV needs a sensitive tool to analyse it. It is concluded that Mazhar-Eslam variability mean frequency, is a better qualitative measure of sensitivity than others. The Rosenstein algorithm presented lower sensitive $M V F$ estimates than the Wolf algorithm to getvariations in local dynamic stability from small data sets. The data confirming the idea that latest outcome observations from the inability and ability of the Wolf algorithm and Rosenstein algorithm, respectively, to estimate adequately MVF of attractors with significant of convergence. Therefore, the Mazhar-Eslam algorithm seems to be more suitable to evaluate local dynamic stability for any data sets especially small one like HRV. When the data set size is raise, it be provided to make the observations of the Mazhar-Eslam algorithm more convenient, although other means as raising the sample size might have a same impact. The Mazhar-Eslam algorithm takes the same strategy of Rosenstein method for initial step to calculate the lag and mean period, but it uses the merits of Discrete Wavelet Transform (DWT) instead of Fats Fourier Transform (FFT) unlike Rosenstein. After that, it completes steps of calculating $\Omega_{M}$ as Wolf method. The Mazhar-Eslam method care of all variants especially the small ones like that are in HRV. These variants may contain many important data to diagnose diseases as R-R interval has many variants. Thus, the Mazhar-Eslam algorithm for MVF $\overline{\Omega_{M}}$ takes all of $\Omega_{M}$ s. That makes it to be robust predictor, that appear in different results among Mazhar-Eslam, Wolf, and Rosenstein. The Mazhar-Eslam algorithm presents a new chapter for HRV prediction. It contains a positive part for HRV as it is stochaotic signal. The introduced Bipolar Mazhar-Eslam MVF supports to follow-up the cases and it has the ability of monitoring. The Bipolar Mazhar-Eslam MVF shows that Mazhar-Eslam algorithm is 
more accurate and sensitive than Wolf and Rosenstein MVF. The next table 4 discusses the sensitivity and accuracy of MVF algorithms as it is clear the Mazher-Eslam is the best MVF algorithm for HRV.

Table 4. SED of MVF algorithms.

\begin{tabular}{|l|l|c|l|c|}
\hline Cases No. & Mazher Eslam $(\mathrm{ME})$ & $\begin{array}{l}\text { Wolf } \\
(\mathrm{W})\end{array}$ & Rosenstein (R) & SED \\
\hline $\begin{array}{l}112,101,124,119 \\
\text { and } 115\end{array}$ & $\begin{array}{l}0.0800,0.0830,0.0840,0.0900, \\
0.0930\end{array}$ & 0.1700 & $\begin{array}{l}0.2400,0.2500,2500,2700, \\
2800\end{array}$ & ME $>\mathrm{W}>\mathrm{R}$ \\
\hline 102,107 and 104 & $0.0530,0.0667,0.0700$ & 0.1300 & $0.1600,0.2000,02100$ & $\mathrm{ME}>\mathrm{W}>\mathrm{R}$ \\
\hline $\begin{array}{l}117,122,111,118 \\
\text { and } 121\end{array}$ & $\begin{array}{l}0.0770,0.0770,0.0800,0.0833, \\
0,0840,\end{array}$ & 0.1600 & $\begin{array}{l}0.2300,0.2300,0.2400,0.2500, \\
0.2500\end{array}$ & ME $>\mathrm{W}>\mathrm{R}$ \\
\hline $\begin{array}{l}212,221,230 \text { and } \\
109\end{array}$ & $0.0700,0.0700,0.0700,0.0733$ & 0.1400 & $0.2100,0.2100,0.2100,02200$ & $\mathrm{ME}>\mathrm{W}>\mathrm{R}$ \\
\hline $\begin{array}{l}231,203,200,123 \\
\text { and } 106\end{array}$ & $\begin{array}{l}0.0740,0.0770,0.0770,0.0770, \\
0.0770\end{array}$ & 0.1500 & $\begin{array}{l}0.2200,0.2300,0.2300,0.2300, \\
0.2300\end{array}$ & ME $>\mathrm{W}>\mathrm{R}$ \\
\hline
\end{tabular}

\section{REFERENCES}

[1] Beckers, Frank, Bart Verheyden, Dirk Ramaekers, Bernard Swynghedauw, And André E. Aubert. "Effects Of Autonomic Blockade On Non-Linear Cardiovascular Variability Indices In Rats." Clinical And Experimental Pharmacology And Physiology 33, No. 5-6 (2006): 431-439.

[2] Reyes Del Paso, Gustavo A., Wolf Langewitz, Lambertus Jm Mulder, Arieroon, And Stefan Duschek. "The Utility Of Low Frequency Heart Rate Variability As An Index Of Sympathetic Cardiac Tone: A Review With Emphasis On A Reanalysis Of Previous Studies." Psychophysiology 50, No. 5 (2013): 477-487.

[3] Zannad, Faiez, Gaetano M. De Ferrari, Anton E. Tuinenburg, David Wright, Josepbrugada, Christian Butter, Helmut Klein Et Al. "Chronic Vagal Stimulation For The Treatment Of Low Ejection Fraction Heart Failure: Results Of The Neural Cardiac Therapy For Heart Failure (Nectar-Hf) Randomized Controlled Trial." European Heart Journal 36, No. 7 (2015): 425-433.

[4] Kobayashi, Hiromitsu, Bum-Jin Park, And Yoshifumi Miyazaki. "Normative References Of Heart Rate Variability And Salivary Alpha-Amylase In A Healthy Young Male Population." J Physiolanthropol 31, No. 9 (2012).

[5] Mazhar B. Tayel And Eslam I Alsaba. Robust And Sensitive Method Of Lyapunov Exponent For Heart Rate Variability. Internationaljournal Of Biomedical Engineering And Science (Ijbes), Vol. 2, No. 3, July 2015. Pp $31-48$

[6] Mazhar B. Tayel And Eslam I Alsaba. A Modified Method For Predictivity Of Heart Rate Variability. Computer Science And Information Technology (Cs\&It) - Cscp 2015. Pp 67 - 77 